\title{
Photodissociation dynamics of the ortho- and para-xylyl radicals
}

\author{
Kai Pachner, ${ }^{1}$ Mathias Steglich, ${ }^{2}$ Patrick Hemberger, ${ }^{2}$ and Ingo Fischer ${ }^{1, a)}$ \\ ${ }^{1}$ Institute of Physical and Theoretical Chemistry, University of Würzburg, Am Hubland, D-97074 Würzburg, \\ Germany \\ ${ }^{2}$ Laboratory for Femtochemistry and Synchrotron Radiation, Paul Scherrer Institut (PSI), CH-5232 Villigen, \\ Switzerland
}

(Received 6 May 2017; accepted 11 August 2017; published online 28 August 2017)

\begin{abstract}
The photodissociation dynamics of the $\mathrm{C}_{8} \mathrm{H}_{9}$ isomers ortho- and para-xylyl are investigated in a free jet. The xylyl radicals are generated by flash pyrolysis from 2-(2-methylphenyl)- and 2-(4methylphenyl) ethyl nitrite and are excited into the $\mathrm{D}_{3}$ state. REMPI- spectra show vibronic structure and the origin of the transition is identified at $32291 \mathrm{~cm}^{-1}$ for the para- and at $32132 \mathrm{~cm}^{-1}$ for the ortho-isomer. Photofragment $\mathrm{H}$-atom action spectra show bands at the same energy and thus confirm $\mathrm{H}$-atom loss from xylyl radicals. To gain further insight into the photodissociation dynamics, velocity map images of the hydrogen atom photofragments are recorded. Their angular distribution is isotropic and the translational energy release is in agreement with a dissociation to products in their electronic ground state. Photodissociation of para-xylyl leads to the formation of para-xylylene $\left(\mathrm{C}_{8} \mathrm{H}_{8}\right)$, while the data for ortho-xylyl agree much better with the isomer benzocyclobutene as the dominant molecular fragment rather than ortho-xylylene. In computations we identified a new pathway for the reaction ortho-xylyl $\rightarrow$ benzocyclobutene $+\mathrm{H}$ with a barrier of $3.39 \mathrm{eV}\left(27340 \mathrm{~cm}^{-1}\right)$, which becomes accessible at the employed excitation energy. It proceeds via a combination of scissoring and rotational motion of the $-\mathrm{CH}_{2}$ and $-\mathrm{CH}_{3}$ groups. However, the observed rate constants measured by delaying the excitation and ionization laser with respect to each other are significantly faster than computed ones, indicating intrinsic non-RRKM behaviour. A comparably high value of around $30 \%$ of the excess energy is released as translation of the $\mathrm{H}$-atom photofragment. Published by AIP Publishing. [http://dx.doi.org/10.1063/1.4999906]
\end{abstract}

\section{INTRODUCTION}

The replacement of benzene as an anti-knock agent in combustion processes by alkylated aromatic hydrocarbons like toluene or xylene has led to increased interest in the chemical reactions of these compounds. ${ }^{1,2}$ The primary reaction under high temperature conditions is hydrogen atom abstraction on the alkyl side chain. However, the radicals formed in this step are resonance stabilized, ${ }^{3}$ exhibit a low reactivity towards oxygen, ${ }^{4}$ are thus long-lived in combustion engines and have a high tendency to form PAHs in bimolecular reactions. Especially the deactivation mechanism of such fuel radicals is of great interest, because it influences the sooting behavior. The competing unimolecular reactions of resonance stabilized radicals (RSR) and their detailed mechanism are therefore of relevance for combustion chemistry. ${ }^{3}$ An often applied laboratory strategy that we also follow in the present work is to study RSR under isolated conditions, deposit a well-defined amount of energy in the reactant by optical excitation and subsequently analyze the unimolecular reaction products by spectroscopic methods. ${ }^{5-7}$ Note that thermal excitation (heating) results in a broad energy distribution, which complicates analysis of the dissociation.

The chemistry of the related benzyl radical, the primary dissociation product of toluene, has been investigated in some

\footnotetext{
a) E-mail: ingo.fischer@uni-wuerzburg.de
}

detail. Several studies aimed at the kinetics of benzyl to determine rate constants at high-temperature, ${ }^{8-13}$ and to identify the decomposition products in a high-temperature reactor similar to the one employed in the present work. ${ }^{14,15}$ The unimolecular dissociation after excitation at $248 \mathrm{~nm}$ was investigated by translational energy spectroscopy ${ }^{16}$ and between 228 and $270 \mathrm{~nm}$ by Rydberg time-of-flight spectroscopy. ${ }^{17}$ $\mathrm{H}$-atom loss leading to fulvenallene has been found to be the dominant reaction pathway in agreement with theory. ${ }^{18-20}$ The nonradiative deactivation after UV excitation was studied by time-resolved spectroscopy and a fast deactivation of the UV excited states through conical intersections was identified. $^{21-23}$

In contrast, work on the three xylyl radical isomers has been limited so far. Only the $\mathrm{D}_{1} \leftarrow \mathrm{D}_{0}$ transition (A ${ }^{2} \mathrm{~A}_{2}$ $\leftarrow \mathrm{X}^{2} \mathrm{~B}_{2}$ in $\mathrm{C}_{2 \mathrm{v}}$ symmetry) has been studied by laser induced fluorescence. ${ }^{24-26}$ Recently all three isomers of xylyl have been selectively generated by pyrolysis and observed via threshold photoelectron spectroscopy. ${ }^{2}$ Ionization energies of $7.08 \mathrm{eV}, 7.11 \mathrm{eV}$, and $6.94 \mathrm{eV}$ have been determined for ortho-, meta-, and para-xylyl, respectively. In addition, their unimolecular decomposition was studied in a microtubular reactor and probed by photoion mass-selected threshold photoelectron spectroscopy. ${ }^{2}$ Reactions of xylyl radicals in a meta-xylene flame have recently been explored by photoionization. ${ }^{27}$ While a number of combustion-relevant high temperature reactions of xylene have been investigated, ${ }^{28-30}$ others were only explored computationally. ${ }^{31-35}$ Xylyl radicals can undergo hydrogen 
loss to form closed shell species, which we summarize below. In contrast to benzyl, which undergoes a ring contraction upon $\mathrm{H}$-atom loss to yield fulvenallene, the six-membered ring can stay intact in ortho- and para-xylyl. The main products will thus be ortho- and para-xylylene, according to (1) and (2)

$$
\text { para-xylyl } \rightarrow \text { para-xylylene }+\mathrm{H}, \quad \Delta_{\mathrm{R}} \mathrm{H}^{\circ}=257.5 \mathrm{~kJ} / \mathrm{mol},
$$

ortho-xylyl $\rightarrow$ ortho-xylylene $+\mathrm{H}, \quad \Delta_{\mathrm{R}} \mathrm{H}^{\circ}=284 \mathrm{~kJ} / \mathrm{mol}$.

The standard heats of reaction, $\Delta_{\mathrm{R}} \mathrm{H}^{\circ}$ were taken from computed $^{33}$ standard heats of formation, $\Delta_{\mathrm{f}} \mathrm{H}^{\circ}$. In Refs. 2 and 36 zero Kelvin values $\Delta_{\mathrm{R}} \mathrm{H}^{\circ}(0 \mathrm{~K})=264.9 \mathrm{~kJ} / \mathrm{mol}$ and $291.2 \mathrm{~kJ} / \mathrm{mol}$ were calculated for (1) and (2), respectively. In addition, other product isomers are possible. H-atom loss from ortho-xylyl can also produce the bicyclic benzocyclobutene (3). The most stable $\mathrm{C}_{8} \mathrm{H}_{8}$ isomer is styrene, which can be formed according to reaction (4):

$$
\begin{aligned}
& \text { ortho-xylyl } \rightarrow \text { benzocyclobutene }+\mathrm{H}, \\
& \qquad \Delta_{\mathrm{R}} \mathrm{H}(0 \mathrm{~K})=245.2 \mathrm{~kJ} / \mathrm{mol}, \\
& \text { ortho-xylyl } \rightarrow \text { styrene }+\mathrm{H}, \\
& \qquad \Delta_{\mathrm{R}} \mathrm{H}(0 \mathrm{~K})=192.0 \mathrm{~kJ} / \mathrm{mol} \text {. }
\end{aligned}
$$

For both reactions only $\Delta_{\mathrm{R}} \mathrm{H}(0 \mathrm{~K})$ have been computed. ${ }^{2}$ However, (4) is associated with a considerable activation energy of $533 \mathrm{~kJ} / \mathrm{mol},{ }^{2}$ and thus becomes accessible only below $224 \mathrm{~nm}$ excitation in single photon processes. The formation of 2- or 3-methylfulvenallene was also investigated computationally: ${ }^{33}$

$$
\begin{aligned}
\text { ortho-xylyl } & \rightarrow \text { 2-methylfulvenallene }+\mathrm{H}, \\
\Delta_{\mathrm{R}} \mathrm{H}^{\circ} & =349 \mathrm{~kJ} / \mathrm{mol}, \\
\text { para-xylyl } & \rightarrow \text { methylfulvenallene }+\mathrm{H}, \\
\Delta_{\mathrm{R}} \mathrm{H}^{\circ} & =352 \mathrm{~kJ} / \mathrm{mol} .
\end{aligned}
$$

The energy difference between 2- and 3-methylfulvallene product is within $1 \mathrm{~kJ} / \mathrm{mol}$ and thus negligible. Furthermore loss of a methyl group has been suggested to be a possible decomposition pathway for xylyl at high temperature: ${ }^{33}$

ortho-xylyl $\rightarrow$ fulvenallene $+\mathrm{CH}_{3}, \quad \Delta_{\mathrm{R}} \mathrm{H}^{\circ}=315 \mathrm{~kJ} / \mathrm{mol}$,

para-xylyl $\rightarrow$ fulvenallene $+\mathrm{CH}_{3}, \quad \Delta_{\mathrm{R}} \mathrm{H}^{\circ}=318 \mathrm{~kJ} / \mathrm{mol}$.

Here we investigate the unimolecular decomposition pathways of two xylyl radical isomers using $\mathrm{H}$-atom photofragment velocity map imaging (VMI) ${ }^{37}$ after UV excitation between 307 and $314 \mathrm{~nm}$ and around $250 \mathrm{~nm}$. Excitation into the wellknown A-band around $450 \mathrm{~nm}$ deposits $\approx 255-260 \mathrm{~kJ} \mathrm{~mol}^{-1}$ in the radicals, which is barely sufficient for the barrierless reaction (1). Reaction (3) is inaccessible at $450 \mathrm{~nm}$, because it is associated with an additional reverse barrier (see below). Vibrational excitation would provide sufficient energy to overcome several of the relevant barriers, but the long fluorescence lifetimes reported by Charlton and Thrush ${ }^{24}$ indicate that the photodissociation probability is small or zero.
Photofragment imaging, in particular VMI allows for determining molecular reaction products and the mechanism by measuring translational energy distributions of the $\mathrm{H}$-atom product with angular resolution. The method is well established for studies in stable molecules ${ }^{38,39}$ and has recently been applied to a number of reactive intermediates, such as propargylene, ${ }^{40}$ cyclopropenylidene, ${ }^{41}$ and ethyl. ${ }^{42}$ With VMI one can deduce information on the molecular reaction products and the reaction mechanism via information on the translational energy distribution and the angular distribution of the $\mathrm{H}$-atom product. Reaction (6) can therefore not be experimentally observed in the present experiment, but based on the computations, the expected branching ratio will be a few percent only. 33

\section{EXPERIMENTAL}

A free jet of ortho- or para-xylyl radicals has been generated by flash pyrolysis ${ }^{5}$ from 2-(2-methylphenyl)ethyl nitrite and 2-(4-methylphenyl)ethyl nitrite, respectively (Fig. 1). The nitrite precursors were prepared from the corresponding 2or 4-methylphenylethanol according to the literature procedure. $^{43}$ Due to the equilibrium between nitrite and alcohol, some methylphenylethanol remained in the reaction mixture. The precursors were heated to about $70^{\circ} \mathrm{C}$, seeded in approximately 2.5 bars Argon and expanded into the vacuum through a pulsed solenoid nozzle at a $10 \mathrm{~Hz}$ repetition rate. An electrically heated $\mathrm{SiC}$ tube was mounted at the exit of the pulsed valve.

The experiments were carried out in a velocity map imaging (VMI) setup. ${ }^{37,38}$ The molecular beam was skimmed and entered into the ion optics through a $5 \mathrm{~mm}$ hole in the repeller plate. Three velocity-focusing plates (repeller, extractor, ground), separated by $15 \mathrm{~mm}$ are used to extract the ions and accelerate them onto the detector using fields of $1013 \mathrm{~V} / \mathrm{cm}$ and $2320 \mathrm{~V} / \mathrm{cm}$. Ions were detected by a dual stage microchannel plate (MCP) detector equipped with a phosphorescent screen (P43). For the purpose of detecting only ions within a selected mass range, the voltage at the back plate of the detector was gated by a push/pull switch with a gate width of $100 \mathrm{~ns}$. Hydrogen atoms were probed in all described VMI experiments. The resulting signal was imaged onto a progressive-scan camera with a $2 / 3^{\prime \prime}$ CCD chip by an achromatic object lens. Each image was accumulated over 10000 laser shots, symmetrized and reconstructed via the pBASEX algorithm, ${ }^{44}$ employing Legendre polynomials up to 2 nd order.

For all VMI experiments, the unfocused frequencydoubled output (0.25-0.5 mJ) of a Nd:YAG laser pumped dye

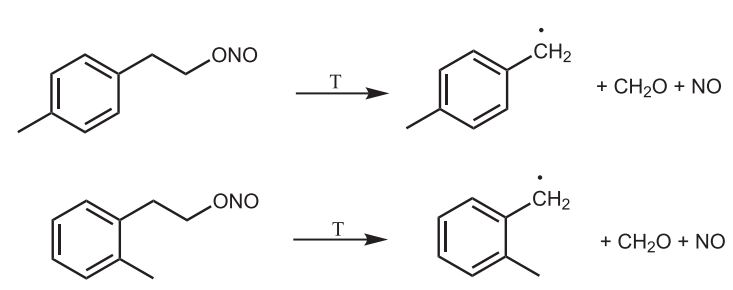

FIG. 1. The para- (upper trace) and ortho- (lower trace) isomers of xylyl are generated by flash pyrolysis of the corresponding nitrites. 
laser was used for excitation. Its polarization was adjusted with a Fresnel double rhomb to be vertically polarized. $\left[1+1^{\prime}\right]$-resonance-enhanced multiphoton ionization (REMPI) was used to detect hydrogen atoms that were formed in a photodissociation event. Therefore, the output (10-13 mJ) of a second frequency-doubled dye laser was focused by a 100 mm lens into a cell filled with 90 Torr of krypton to produce VUV radiation tunable around $121.6 \mathrm{~nm}$ (Lyman- $\alpha$ ). The VUV light was focused into the ionization region by a $100 \mathrm{~mm} \mathrm{MgF}_{2}$ lens mounted at the exit of the cell. Hydrogen atoms are excited from the ${ }^{2} \mathrm{~S}_{1 / 2}$ ground state to the ${ }^{2} \mathrm{P}_{1 / 2,3 / 2}$ states by the absorption at $121.6 \mathrm{~nm}$. The residual fundamental then ionizes the excited atoms. The laser was scanned over $\pm 6 \mathrm{~cm}^{-1}$ around the Lyman- $\alpha$ wavenumber to cover the whole Doppler profile of the $\mathrm{H}$-atoms. In most experiments described below, the ionization laser was delayed by $50 \mathrm{~ns}$ with respect to the excitation laser. The two laser systems were synchronized electronically within $1 \mathrm{~ns}$. The excitation and ionization laser were polarized perpendicularly to each other. A background image was recorded without the excitation laser and subtracted from the two-color image to minimize contributions from onecolor processes. [1+1]-REMPI scans of the xylyl radicals were performed with the excitation laser using significantly higher output power (1.5-3 mJ).

The time of flight (TOF) mass spectra were recorded by monitoring the current at the back plate of the MCP ion detector. TOF mass spectra were recorded at $121.6 \mathrm{~nm}$ and at 118 $\mathrm{nm}$ (generated by frequency-tripling in 17 Torr Xe), because the latter exhibits a better detection efficiency.

\section{RESULTS}

\section{Mass spectra}

In a first step we optimized the experimental conditions, including radical generation in the pyrolysis tube, by photoionization mass spectrometry. Typical mass spectra of para-xylyl and its precursor are given in Fig. 2. Note that the intensity scale is different in the bottom trace. Without pyrolysis and both excitation $(309.7 \mathrm{~nm})$ and ionization $(121.6 \mathrm{~nm})$ laser present four peaks are visible in the mass spectrum (top trace): beside the nitrite precursor $(\mathrm{m} / \mathrm{z}=165)$ a xylyl signal is present that originates most likely from dissociative photoionization of the precursor. This process also produces a small $\mathrm{H}$-atom background signal. In addition, a peak at $\mathrm{m} / \mathrm{z}=136$ is observed that corresponds to the residual alcohol precursor employed in the nitrite synthesis. With pyrolysis turned on and only the ionization laser present (center trace), the mass spectrum looks rather similar and again a small $\mathrm{H}$-atom background signal is seen. The bottom trace finally shows that $\mathrm{H}$-atoms are efficiently formed, when the pyrolysis is turned on and both lasers are present. The $\mathrm{H}$-atom signal increases by a factor of roughly 10 compared with the background signal in the top and center trace, thus most $\mathrm{H}$-atoms originate from the photodissociation of para-xylyl rather than from background. Note that the nitrite precursor is not fully converted under these conditions.

Mass spectra of the ortho-compound look very similar and are thus given only in the supplementary material (see Fig. S1).

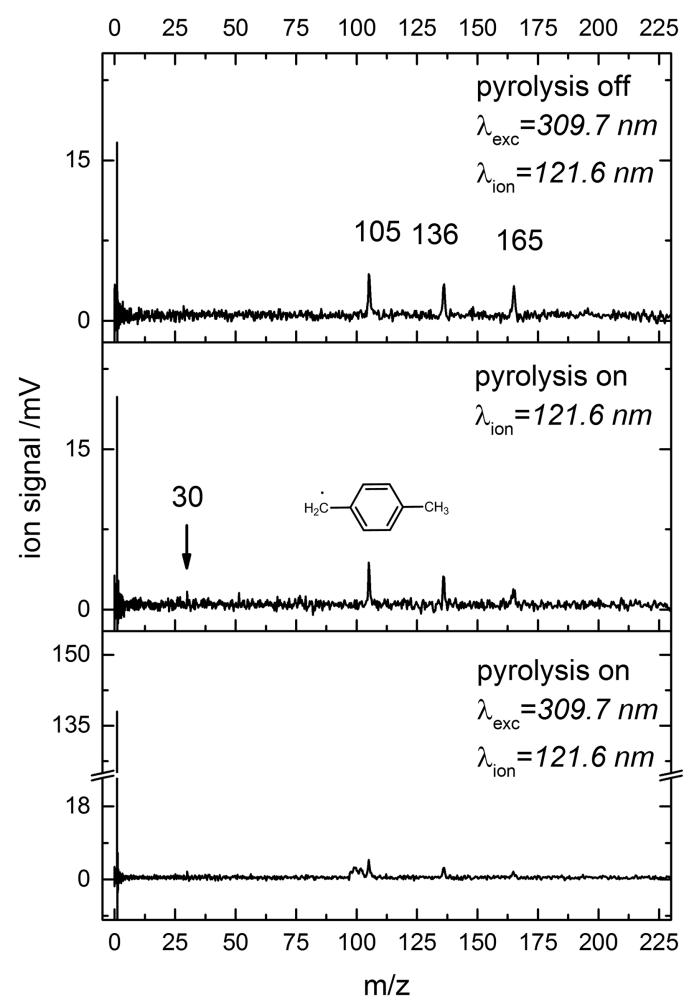

FIG. 2. Mass spectra of 2-(4-methylphenyl)ethyl nitrite $(\mathrm{m} / \mathrm{z}=165)$ under various conditions. A strong hydrogen signal was only observed when both lasers are present. Note the different intensity scale in the bottom trace. A small signal at $\mathrm{m} / \mathrm{z}=30$ is present when the pyrolysis is turned on (center trace).

\section{REMPI- and H-atom action spectra}

Vibronically resolved emission spectra of the $\mathrm{D}_{1} \leftarrow \mathrm{D}_{0}$ transitions around 450-500 $\mathrm{nm}$ have been recorded for all three isomers of xylyl. ${ }^{24-26,45}$ In contrast experimental and computational knowledge on the higher electronically excited states of xylyl radicals is scarce. As the electronic structure can be expected to be similar to one of the benzyl radicals, the transitions of the latter provide a good starting point for a search. The origin of the $\mathrm{D}_{3} \leftarrow \mathrm{D}_{0}$ transition in benzyl was observed previously at $305.16 \mathrm{~nm} .^{23,46,47}$ Although originally assigned to a $\pi \pi^{*}$ excitation, the $\mathrm{D}_{3}$ state was recently reassigned to a $\mathrm{C}^{2} \mathrm{~A}_{1} 3 \mathrm{~s}$ Rydberg state. ${ }^{22}$ In benzyl, a correct assignment required multi-reference configuration interaction (MRCI) calculations with a large basis set; an approach that is beyond our means in the present work. We therefore assume that the description obtained for benzyl also holds for both xylyl isomers. In fact, vibronic transitions of xylyl can be observed in this region as shown in Fig. 3: In the REMPI-spectrum of para-xylyl (black line, upper trace of Fig. 3) an intense band is identified at $32291(309.7 \mathrm{~nm})$ and assigned to the $\mathrm{D}_{3}$ origin. The standard deviation of $\pm 15 \mathrm{~cm}^{-1}$ is estimated from the half width at half maximum of the band. A second band, red-shifted by $-86 \mathrm{~cm}^{-1}$ is due to a hot-band or sequence-band transition. In contrast, the REMPI-spectrum of ortho-xylyl (black line, lower trace of Fig. 3) shows more structure. The band at $32132 \mathrm{~cm}^{-1}(311.2 \mathrm{~nm})$ corresponds probably to the origin of the transition. In addition, several smaller bands appear: While the two bands at +138 and $+210 \mathrm{~cm}^{-1}$ are due to lowenergy bending vibrations, the structure on the blue side of 


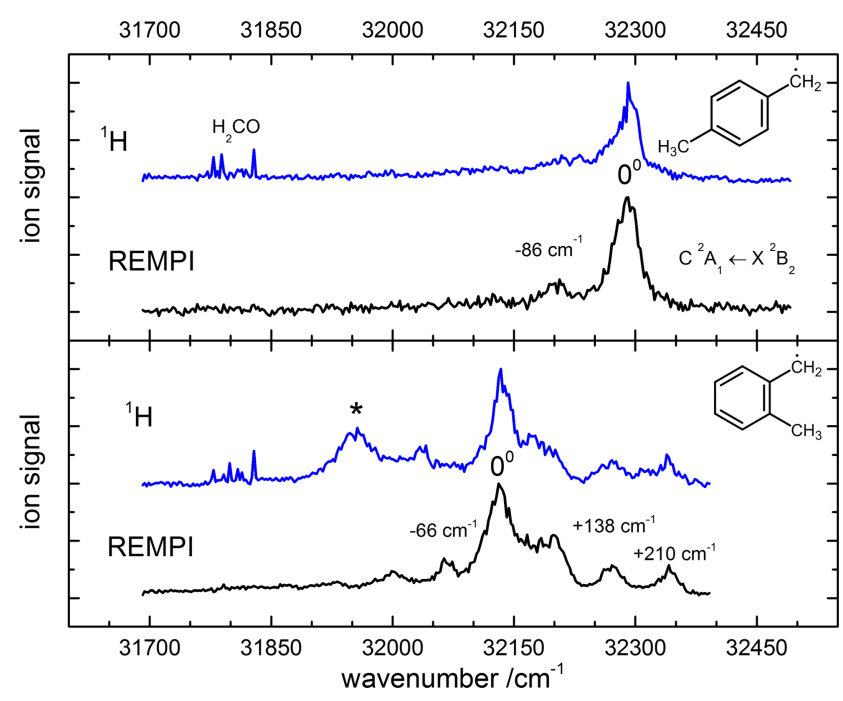

FIG. 3. The resonant MPI spectrum of the xylyl radicals and the H-atom action spectrum of the hydrogen atom fragments recorded as a function of the excitation wavelength. For both para- (upper trace) and ortho-xylyl (lower trace) resonances are visible. In the $\mathrm{H}$-atom action spectrum of ortho-xylyl an additional peak is present around $31960 \mathrm{~cm}^{-1}$ that is not visible in the REMPI-spectrum (asterisk).

the origin band can be assigned to torsional transitions of the methyl rotor. Recent theoretical work computed a barrier for internal rotation of $1.9 \mathrm{kcal} / \mathrm{mol}(82 \mathrm{meV})$ for ortho-xylyl, but only $0.01 \mathrm{kcal} / \mathrm{mol}(0.4 \mathrm{meV})$ for the six-fold barrier in paraxylyl. ${ }^{33}$ Assuming comparable barriers in the excited state, it is therefore not surprising that discrete torsional transitions appear only in the spectrum of ortho-xylyl. With respect to the benzyl $\mathrm{D}_{3}$ origin at $305.16 \mathrm{~nm}$, the transitions in xylyl are redshifted by approximately $-470 \mathrm{~cm}^{-1}$ (para) and $-630 \mathrm{~cm}^{-1}$ (ortho). The spectra of benzyl and para-xylyl have a similar appearance with little activity in low-wavenumber vibrations, but the width of the origin band appears narrower in para-xylyl.

In benzyl, a lifetime of $400 \mathrm{fs}$ was measured for the $\mathrm{D}_{3}$ origin, ${ }^{23}$ therefore rotational resolution cannot be expected. However radicals are formed with considerable rotational excitation in the pyrolysis, so a fit of the band contour can provide temperature information. Band contour fits using the pgopher program ${ }^{48}$ are shown in the supplementary material [see Fig. S2 (para) and Figure S3 (ortho)]. They yield a rotational temperature of around $40 \mathrm{~K}$ for both radicals. A more detailed analysis of the electronic spectrum is currently under way.

In addition to the REMPI spectra we also recorded the $\mathrm{H}$-atom photofragment signal by scanning the excitation laser in the presence of the $121.6 \mathrm{~nm}$ ionization laser. The resulting $\mathrm{H}$-atom action spectra are given as blue lines in Fig. 3. All peaks discernible in the REMPI-spectra also appear in the $\mathrm{H}$-atom action spectra, indicating that the $\mathrm{H}$-atom signal at these excitation wavelengths originates indeed from xylyl. Between $31778 \mathrm{~cm}^{-1}$ and $31828 \mathrm{~cm}^{-1}$ a series of sharp lines can be recognized in both spectra. These lines originate from the transition into the $\mathrm{A}^{1} \mathrm{~A}_{2}\left(2^{3} 4^{1}\right)$ state of formaldehyde, ${ }^{49,50}$ which is produced as a side product during pyrolysis (cf. Fig. 1). Although $\mathrm{CO}+\mathrm{H}_{2}$ is the dominant photofragmentation pathway in $\mathrm{CH}_{2} \mathrm{O}$, a minor one leading to $\mathrm{HCO}+\mathrm{H}$, has been detected before above $30300 \mathrm{~cm}^{-1} .{ }^{51}$ Furthermore one band appears in the $\mathrm{H}$-atom action spectrum at $31953 \mathrm{~cm}^{-1}$, marked with an asterisk in Fig. 3 that does not show up in the REMPI-spectrum of ortho-xylyl. An H-atom signal was always absent if only the precursor (without pyrolysis) or the 2-(2-methylphenyl) ethanol (residual contamination from synthesis) were used. Formaldehyde, $\mathrm{CH}_{2} \mathrm{O}$, can be also excluded, because the band is only found in the spectrum of ortho-xylyl. Since a mass spectrum at $31953 \mathrm{~cm}^{-1}$ does not reveal any additional peaks, we assign this band to a pyrolysis side product that can be excited around $310 \mathrm{~nm}$, but not ionized with $10.196 \mathrm{eV}$ (Lyman- $\alpha$ radiation).

\section{PHOTOFRAGMENT IMAGING EXPERIMENTS}

\section{Para-xylyl}

The obvious photodissociation product associated with $\mathrm{H}$-atom loss is para-xylylene depicted in Fig. 4. To gain further insight into the dynamics of the dissociation process, we excited the molecule into the presumed $\mathrm{D}_{3}$ origin at $32291 \mathrm{~cm}^{-1}$ and recorded velocity map images of the $\mathrm{H}-$ atom photofragment at a laser power of $0.25 \mathrm{~mJ}$. As visible from Fig. S4 of the supplementary material the signal depends linearly on the laser power in this region. From the image, which appeared isotropic, the translational energy distribution, $P\left(E_{T}\right)$ shown in Fig. 4 was obtained. It gives the probability of a certain translational energy release (TER) taking place in the experiment. It peaks at around $0.3 \mathrm{eV}$, which corresponds to $29 \mathrm{~kJ} \mathrm{~mol}^{-1}$. For the fit we assumed the functional form $^{52}$

$$
P(f T)=f T^{a} \times(1-f T)^{b}
$$

with $f T$ being the fraction of excess energy (rather than the absolute amount) being released as translation, and $a$ and $b$ being adjustable parameters. The resulting fit to our data is described by the solid blue line in Fig. 4. An expectation value $\left\langle E_{T}\right\rangle=0.41 \mathrm{eV}$ of the $\mathrm{H}$-atom translational energy release is computed, which corresponds to a fraction $\left\langle f_{T}\right\rangle=0.33$, i.e.,

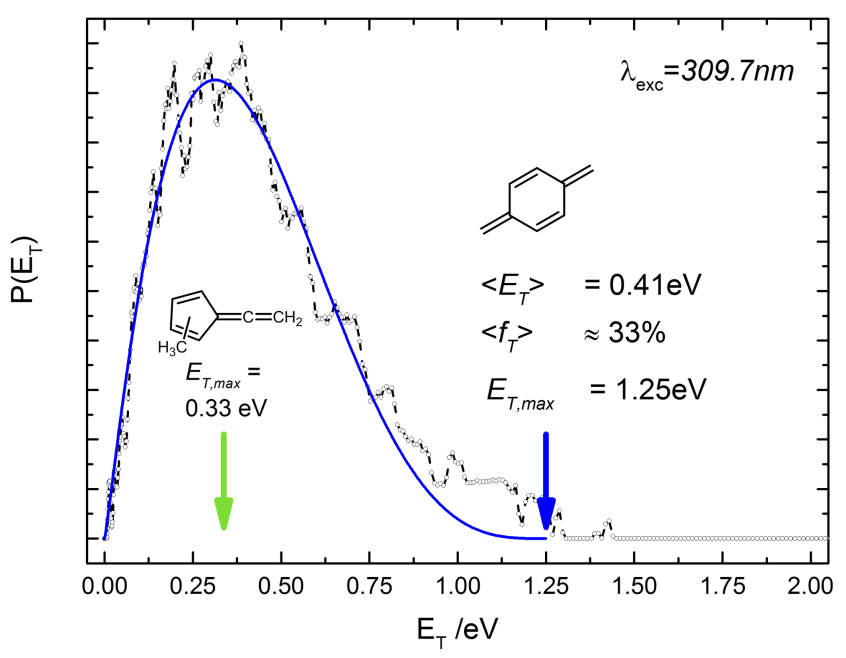

FIG. 4. The H-atom photoproduct translational energy distribution of paraxylyl, derived from the velocity map image, together with a fit obtained by forward convolution (see text). Para-xylyl was excited at $32291 \mathrm{~cm}^{-1}$ with a laser power of $0.25 \mathrm{~mJ}$. $E_{\mathrm{T} \text {,max }}$ represents the maximum translational energy release for a given product. The expectation values given in the figure correspond to para-xylylene as the molecular fragment. 
$33 \%$ of the total excess energy being released as translation. Although this is larger than the release observed for hydrocarbon radicals like allyl and propargyl, where around $20 \%$ were found, ${ }^{5}$ the experimental $P\left(E_{\mathrm{T}}\right)$ is concomitant with a photodissociation according to reaction (1) that proceeds on the ground state potential energy surface. The expected dissociation product is para-xylylene. With the computed BDE of 265 $\mathrm{kJ} \cdot \mathrm{mol}^{-1}$ we calculate a maximum translational energy release (TER) of $E_{\mathrm{T}, \max }=121 \mathrm{~kJ} \mathrm{~mol}^{-1}$ or $1.25 \mathrm{eV}$ for this pathway. An alternative product is 2- or 3-methylfulvenallene (5b), but $E_{\text {T,max }}$ would be $0.33 \mathrm{eV}$ (green arrow in Figure 4), in disagreement with the measured TER. As visible at $E_{T}>1 \mathrm{eV}$ the data points are systematically above the fit line. When the excitation laser power was increased to $0.5 \mathrm{~mJ}$, the signal/noise ratio of the data improved, but the contribution at high $E_{T}$ became more pronounced. Fast $\mathrm{H}$-atoms originate therefore most likely from multiphoton excitation processes, despite the linear power dependence of the total signal (see Figs. S4 and S5 of the supplementary material for power dependence of $\mathrm{H}$-atom signal from para- and ortho-xylyl).

The angular distribution $P(\theta)$ of the $\mathrm{H}$-atom photofragments is depicted in Fig. 5. The solid line represents a fit to the data using the standard recoil anisotropy function

$$
P(\theta)=\frac{1}{4 \pi}\left[1+\beta \times P_{2}(\cos \theta)\right]
$$

with $P_{2}$ being the second order Legendre polynomial and $\beta$ the anisotropy parameter that is optimized in the fit. There is no appreciable anisotropy for the $\mathrm{H}$-atoms with low $E_{T}$ and a $\beta$-parameter of around 0 is obtained. However, analysis of the H-atoms with $E_{T}>0.98 \mathrm{eV}$ yields $\beta \approx-0.63$ (see Fig. S6 of the supplementary material), indicating an additional contribution of fast anisotropic $\mathrm{H}$-atoms. This confirms that the small part of the H-atom signal above approximately $1 \mathrm{eV}$ in Fig. 4 originates from multiphoton excitation processes.

We also carried out experiments at shorter wavelengths around $250 \mathrm{~nm}$ (see Fig. 6). By comparison with benzyl para-xylyl is excited into the D-band, where most of the oscillator strength is contributed by a $\pi \pi^{*}$ transition. No

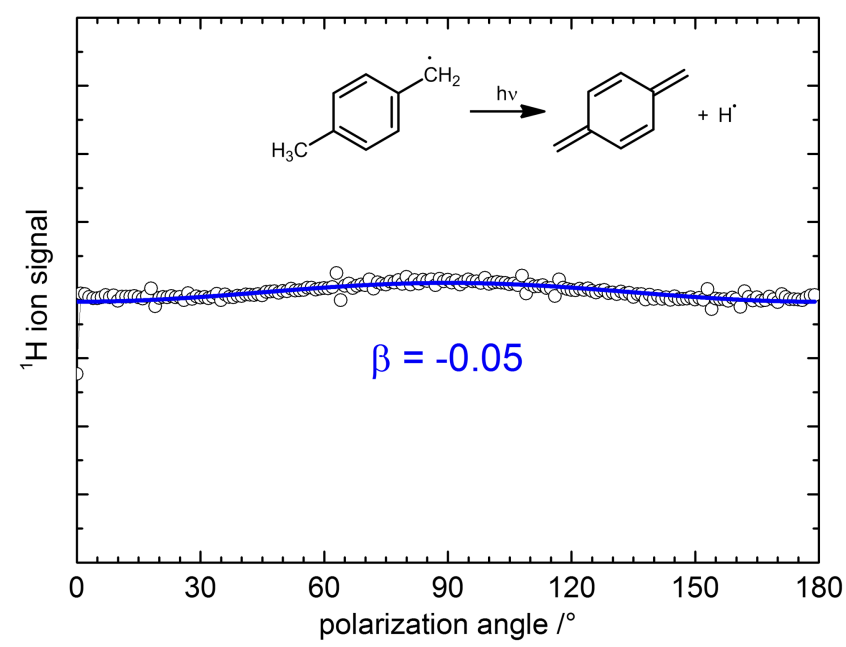

FIG. 5. An isotropic photofragment angular distribution is observed for $\mathrm{H}$ atoms from para-xylyl at $309.7 \mathrm{~nm}$ excitation (signal averaged overall $E_{T}$ ).

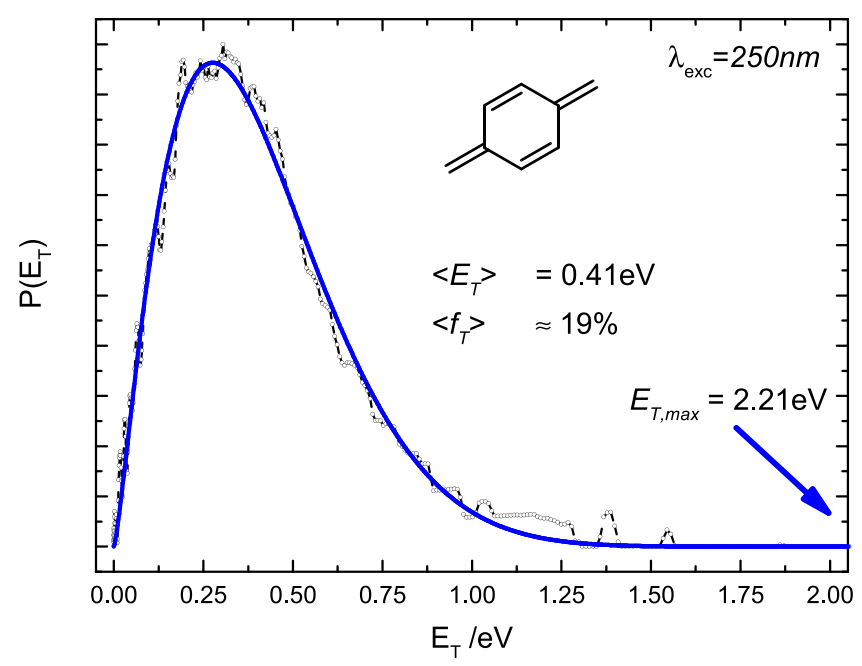

FIG. 6. The H-atom photoproduct translational energy distribution of paraxylyl excited at $250 \mathrm{~nm}$. $E_{\mathrm{T} \text {,max }}$ represents the maximum translational energy release for the para-xylylene product.

structured REMPI- or H-atom action spectrum could be recorded, but control experiments revealed no appreciable $\mathrm{H}$-atom signal from either the precursor or the alcohol. Therefore, we are confident that para-xylyl is the dominant $\mathrm{H}$ atom source at $250 \mathrm{~nm}$. Again, an isotropic H-atom PAD is observed and $\left\langle f_{T}\right\rangle$ decreases to $\left\langle f_{T}\right\rangle=0.19$, assuming reaction (1). The TER is in full agreement with a statistical process.

\section{Ortho-xylyl}

In contrast to the para-xylyl case at least four molecular fragments are possible in an $\mathrm{H}$-atom loss reaction from ortho-xylyl, ortho-xylylene (2), benzocyclobutene (3), styrene (4), and methylfulvenallene (5a); see Fig. 7 for the chemical structures. Based on the reaction pathways and activation barriers computed by da Silva et al. only ortho-xylylene should be accessible at $32132 \mathrm{~cm}^{-1}(3.98 \mathrm{eV} / 311.2 \mathrm{~nm})$. Benzocyclobutene is thought to be formed via TS3a at $4.19 \mathrm{eV}$ and constitutes a [2+2] cycloaddition after $\mathrm{H}$-atom loss to the intermediately formed $o$-xylylene. Styrene is the thermodynamically preferred product, but is generated in a multisteppathway that proceeds via a carbene intermediate with a large

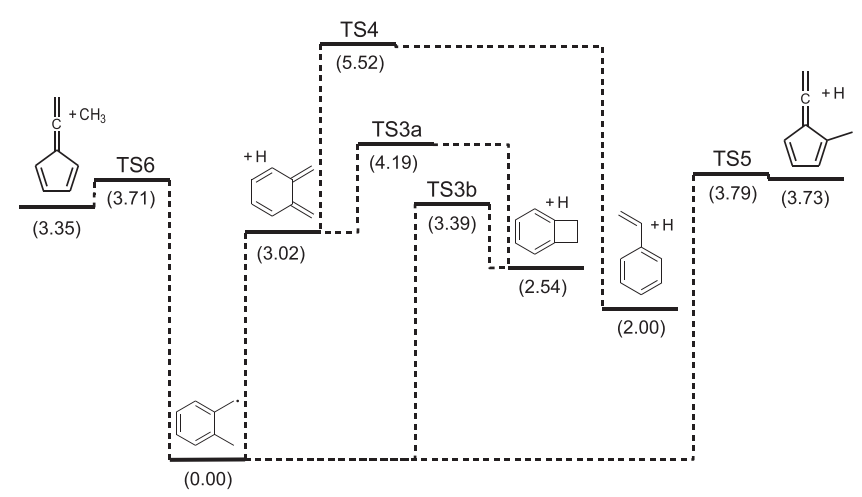

FIG. 7. Simplified reaction coordinates for the pathways to products from ortho-xylyl. Note that for more complex reaction pathways only the highestlying transition state is indicated in the figure. Energies are given in $\mathrm{eV}$ and are taken from Refs. 2, 33, and 36. 


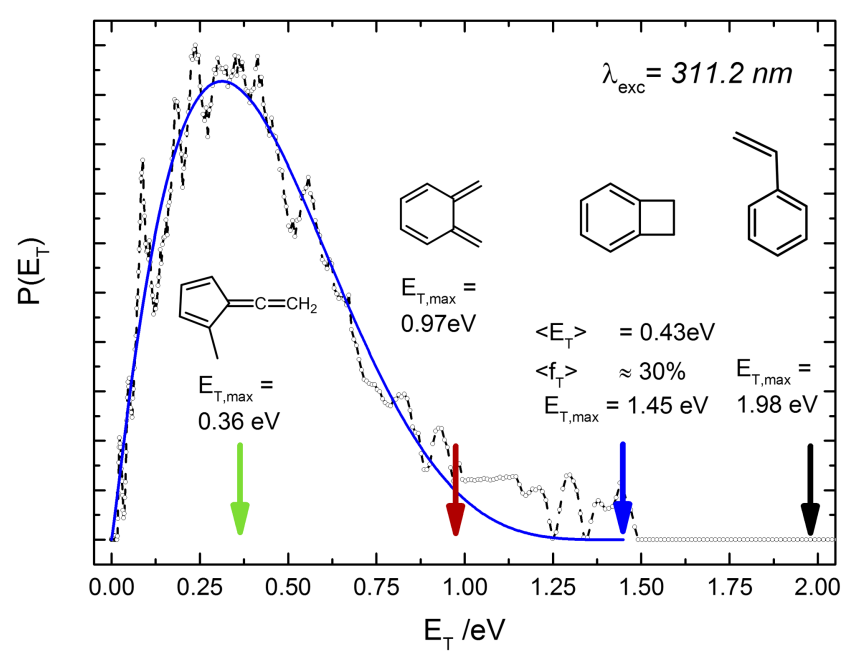

FIG. 8. The H-atom photoproduct translational energy distribution of orthoxylyl, derived from the velocity map image, together with a fit obtained by forward convolution (blue line). $E_{\mathrm{T}, \max }$ represents the maximum translational energy release for a given product.

activation barrier of $5.52 \mathrm{eV}$ (TS4), more than $1.5 \mathrm{eV}$ higher than the excitation energy employed. To investigate the photodissociation dynamics of ortho-xylyl, we excited the radical into the band at $32132 \mathrm{~cm}^{-1}(311.2 \mathrm{~nm})$ and carried out $\mathrm{H}$ atom VMI experiments. The translational energy distribution, $P\left(E_{T}\right)$ of the $\mathrm{H}$-atom photoproduct is given in Fig. 8, the photofragment angular distribution (PAD) in Fig. 9. Like in the case of para-xylyl, the PAD is isotropic with $\beta=-0.03$ and $P\left(E_{T}\right)$ is peaked at low energies. Thus, the data hint again at a dissociation that occurs slower than a rotational period from the electronic ground state. We also find a deviation of the data points from the fit at high translational energies, which can again be traced back to multiphoton excitation. The deviation could be reduced by lowering the excitation laser power and the PAD for $\mathrm{H}$-atoms with $\mathrm{E}_{\mathrm{T}}>0.95$ show a significant anisotropy (see Fig. S7 of the supplementary material).

Nevertheless, the interpretation of the TER is not straightforward. While for ortho-xylylene formation an $E_{\mathrm{T}, \max }=0.97$ is expected (red arrow), $P\left(E_{\mathrm{T}}\right)$ is significantly above zero in a translational energy range that is associated with isotropic

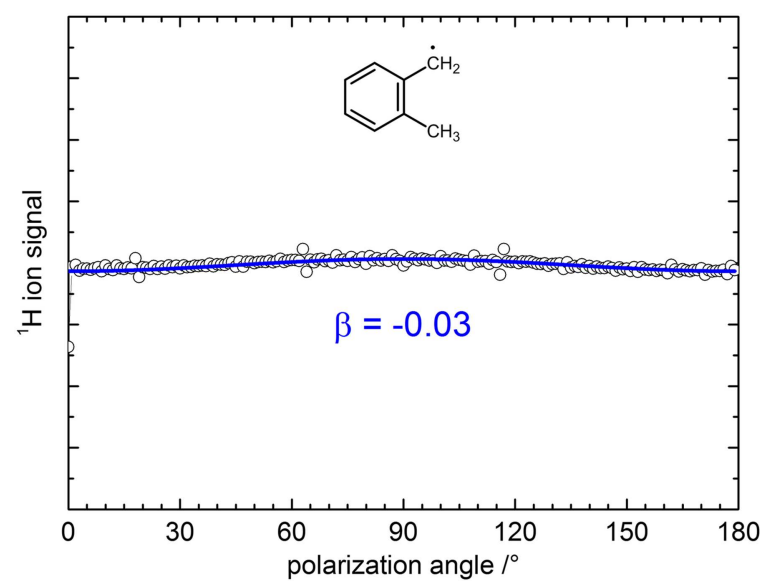

FIG. 9. An isotropic photofragment angular distribution is observed for $\mathrm{H}-$ atoms from ortho-xylyl (averaged overall $E_{T}$ ).
H-atoms (see Fig. S8 of the supplementary material for a best fit of the TER for ortho-xylylene formation). Furthermore, the fraction of the excess energy released into translation of the $\mathrm{H}$-atom would be $\left\langle f_{T}\right\rangle=0.43$, a value outside the confidence range expected for a ground-state dissociation with the observed isotropic PAD. The methylfulvenallene isomer (5a) with $E_{\mathrm{T}, \max }=0.36 \mathrm{eV}$ (green arrow) agrees even less with the measured $P\left(E_{\mathrm{T}}\right)$. Formation of benzocyclobutene on the other hand would match the data much better and yield $\left\langle E_{\mathrm{T}}\right\rangle=0.43 \mathrm{eV}$ with $\left\langle f_{T}\right\rangle=0.30$ and $E_{\mathrm{T}, \max }=1.45 \mathrm{eV}$ (blue arrow). Styrene formation with $E_{\mathrm{T}, \max }=1.98 \mathrm{eV}$ (black arrow) and $\left\langle f_{T}\right\rangle=0.21$ appears to be in agreement with $P\left(E_{\mathrm{T}}\right)$, but as mentioned above the process that includes migration of a $\mathrm{CH}_{2}$ group, has a high barrier of more than $1.5 \mathrm{eV}$ above the photon energy (TS4) and is not accessible at $311.2 \mathrm{~nm}$ within a single photon process. Since in the previously computed pathway to benzocyclobutene the barrier is too high and in any case the initial product ortho-xylylene should determine the characteristics of $\mathrm{H}$-atom loss, we decided to reinvestigate the reaction to benzocyclobutene for a direct pathway from ortho-xylyl to benzocyclobutene without going through the intermediate ortho-xylylene. The calculations were performed with Gaussian $09 .{ }^{53}$ A one-dimensional relaxed potential energy surface scan using density functional theory [DFT, B3LYP//6-311++G(d,p)] was performed by scanning the distance between both methyl groups. The scan helped to identify a second pathway for the formation of benzocyclobutene in which ring formation as well as the loss of the hydrogen atom occur simultaneously. The process proceeds via a scissoring motion of the $-\mathrm{CH}_{2}$ and $-\mathrm{CH}_{3}$ group, combined with a rotational motion of the $\mathrm{CH}_{2}$ group. In the next step the geometry of the transition state was optimized using DFT [B3LYP//6$311++\mathrm{G}(2 \mathrm{df}, \mathrm{p})]$ yielding TS3b with the structure given in the supplementary material (Fig. S10), followed by an energy optimization using the $\mathrm{G} 4$ approach. We have thus indeed found a reaction pathway from ortho-xylyl to benzocyclobutene with an initial barrier of $3.39 \mathrm{eV}$ (TS3b, Fig. 7), without going through a xylylene intermediate. It is accessible at excitation wavelengths around $310 \mathrm{~nm}$, in agreement with $P\left(E_{\mathrm{T}}\right)$. The coordinates of the stationary points are given in the supplementary material. The fit to the TER based on this pathway is given as a blue line in Fig. 8 and matches the measured distribution quite well. $\mathrm{H}$-atoms from (2) will certainly contribute to the TER, but a simultaneous fit is ambiguous due to the number of free parameters.

We also excited ortho-xylyl at $250 \mathrm{~nm}(4.96 \mathrm{eV})$ and recorded $\mathrm{H}$-atom velocity map images. Assuming reaction (3) to proceed via TS3b for $\left\langle f_{T}\right\rangle$ a value of $\left\langle f_{T}\right\rangle=0.16$ and an isotropic PAD are obtained, suggesting a statistical dissociation (see Fig. S9 of the supplementary material). However, the data are difficult to interpret because reaction pathways (2), (3), and (5a) are energetically accessible while (4) remains inaccessible, and reaction (3) can proceed either directly via TS3b or sequentially via TS3a. A further complication arises from the peak at $31953 \mathrm{~cm}^{-1}$ in the $\mathrm{H}$ atom action spectrum (Fig. 3), which indicates the presence of a yet unidentified reactant. In the region around $311 \mathrm{~nm}$ where the REMPI-spectrum is structured, ortho-xylyl can be selectively excited, but this is not the case at $250 \mathrm{~nm}$. We 
therefore consider the analysis of ortho-xylyl at $250 \mathrm{~nm}$ to be tentative.

In addition to the thermochemistry, the reaction kinetics has also to be considered. Since the barrier to benzocyclobutene via TS3b $(3.39 \mathrm{eV})$ is higher than the one to ortho-xylylene $(3.02 \mathrm{eV})$ and the transition state is rather tight, a slower reaction rate can be expected. We therefore carried out simple RRKM calculations to compute unimolecular rates for all reactions. For better comparison, product wavenumbers were used and augmented by two modes with $100 \mathrm{~cm}^{-1}$ and $50 \mathrm{~cm}^{-1}$ to compensate for the lost translational modes (see the supplementary material for a list of vibrational wavenumbers). In fact, for reaction to ortho-xylylene $\mathrm{k}_{311 \mathrm{~nm}}$ $=1.8 \times 10^{3} \mathrm{~s}^{-1}$ and $\mathrm{k}_{250 \mathrm{~nm}}=1.2 \times 10^{6} \mathrm{~s}^{-1}$ were obtained for excitation at $311.2 \mathrm{~nm}$ and $250 \mathrm{~nm}$, respectively, while for the pathway to benzocyclobutene via TS3b $\mathrm{k}_{311 \mathrm{~nm}}=5$ $\times 10^{-1} \mathrm{~s}^{-1}$ and $\mathrm{k}_{250 \mathrm{~nm}}=2.5 \times 10^{3} \mathrm{~s}^{-1}$ were computed, several orders of magnitude slower than for reaction (2). Employing the transition state frequencies for the pathway to benzocyclobutene (3b) rate constants of $\mathrm{k}_{311 \mathrm{~nm}}=3 \times 10^{-2} \mathrm{~s}^{-1}$ and $\mathrm{k}_{250 \mathrm{~nm}}=1.2 \times 10^{2} \mathrm{~s}^{-1}$ were calculated, i.e., the chosen frequencies have only a small influence. Note that the torsional motion of the methyl rotor in xylyl was treated as a vibration, but this should only have a negligible effect on the relative rates. Thus, based on kinetic considerations, formation of benzocyclobutene via TS3b cannot compete with the hydrogen loss reaction to yield ortho-xylylene described by statistical theories.

For comparison, we also measured reaction rates by delaying the two lasers with respect to each other. The rate constant for $\mathrm{H}$-atom loss is then derived from the rise time of the $\mathrm{H}$-atom ion signal, while the decay is due to motion of the hydrogen atoms out of the observation region. ${ }^{6}$ A typical time-delay scan recorded at $311.2 \mathrm{~nm}$ is given in Fig. 10. It shows a rate constant, derived from the rising edge of the signal, in the range of $\mathrm{k}_{H}=10^{8} \mathrm{~s}^{-1}$, which is close to or faster than the instrument function defined by the ns pulse duration of the lasers. For $\mathrm{H}$ loss from para-xylyl, reaction (1) $\mathrm{k}_{309 \mathrm{~nm}}=9.5 \times 10^{3} \mathrm{~s}^{-1}$ and $\mathrm{k}_{250 \mathrm{~nm}}=1.5 \times 10^{6} \mathrm{~s}^{-1}$ were computed, while $\mathrm{k}_{H}$ was again on the order of $10^{8} \mathrm{~s}^{-1}$. Eliminating the lowest wavenumber mode (i.e., the torsion in the reactant) increased $\mathrm{k}_{309 \mathrm{~nm}}$ by a factor of

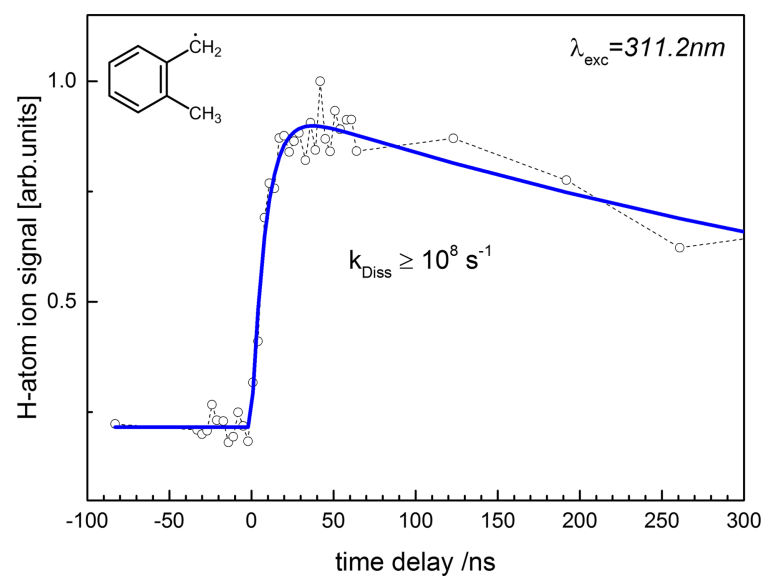

FIG. 10. H-atom time-delay scans for the hydrogen loss from ortho-xylyl at $311.2 \mathrm{~nm}$. As visible the signal rise time is on the order of $10 \mathrm{~ns}$, close to or shorter than the time resolution of the setup.
4. In the translational energy distributions (cf. Fig. 4) shown above, a small contribution of multi-photon excitation is evident, but it is not expected to dominate the time-delay scans. Even if the crude treatment of the methyl rotor is considered, the computed rates around $310 \mathrm{~nm}$ do not describe the experimental rate constants even approximately and the $\mathrm{H}$-atom loss from both radicals is several orders of magnitude faster than expected from RRKM-computations. Interestingly, a similar observation was reported by Song et al. in their work on benzyl. ${ }^{17}$ They measured rates for $\mathrm{H}$-atom loss around $250 \mathrm{~nm}$ that were roughly two orders of magnitude faster than those computed by RRKM. This indicates a common underlying reason for the deviation.

\section{DISCUSSION}

The experiments described above reveal information on the hitherto unexplored electronic structure and photochemistry of the ortho- and para-xylyl radicals after UV excitation. The results obtained can be rationalized by comparison with the more extensively studied benzyl radical, because the electronic structure of the two is expected to be similar. UV light around 309-311 nm excites ortho- and para-xylyl into the $\mathrm{D}_{3}$ state, with the origin slightly red-shifted as compared with benzyl. We assume that recent computations, which assign the $\mathrm{D}_{3}$ state of benzyl to have $3 s$ Rydberg character, ${ }^{22}$ also hold for the xylyl radicals. Around $250 \mathrm{~nm}$ the radicals are excited into the D-band. Here the oscillator strength is contributed in benzyl not only by a strong $\pi \pi^{*}$ transition but also by $p$-Rydberg states. One should keep in mind though that the additional methyl rotor complicates the electronic spectrum due to torsional transitions, as visible in the REMPIspectrum of ortho-xylyl. It also opens a pathway to $\mathrm{H}$-atom loss at the exocyclic methyl group, keeping the six-membered ring intact and forming xylylenes. Furthermore, it increases the density of states, which is of relevance for a description of the deactivation and in the statistical modelling of the dissociation.

Photodissociation of para-xylyl yields para-xylylene, a resonance stabilized biradicaloid ${ }^{54}$ according to (1) via a barrier of only $2.75 \mathrm{eV}(265 \mathrm{~kJ} / \mathrm{mol})$. No other product is expected to compete and in fact the $\mathrm{H}$-atom PAD as well as the recorded translational energy distribution are at all excitation wavelengths in agreement with reaction (1) proceeding from hot ground state molecules. For ortho-xylyl the situation turned out to be more complicated, because a reaction assuming ortho-xylylene as the molecular photofragment does not match the $P\left(E_{\mathrm{T}}\right)$ well. We have computationally identified a direct pathway to benzocyclobutene without going through a xylylene intermediate, which fits the experimental data a lot better. This pathway proceeds by a simultaneous ring formation and hydrogen dissociation via a combination of a scissoring motion of the $\mathrm{CH}_{2}$ and $\mathrm{CH}_{3}$ group and a rotational motion of the $\mathrm{CH}_{2}$ group. Therefore, we assume that pathway (3), again via hot ground state molecules, dominates the dissociation of orthoxylyl and is associated with $\mathrm{H}$-atoms of high $E_{\mathrm{T}}$, despite being kinetically disfavored compared with reaction (2). However, ortho-xylylene will most likely also be formed. A dissociation into the electronic ground state as assumed for both radicals 
requires fast internal conversion. Although no time-resolved data are yet available for xylyl, a fast excited state deactivation is assumed, as it was found for benzyl. Since the density of states is increased in xylyl radicals due to the additional methyl rotor, lifetimes might be even shorter than the density of states in benzyl.

This seemingly simple picture is appealing, but two observations are nevertheless striking. First, rather high translational energy releases are observed in the experiments. For example, photodissociation of para-xylyl at $309.7 \mathrm{~nm}$ is associated with $33 \%$ of the excess energy being released as translation. The value decreases to $19 \%$ at $250 \mathrm{~nm}$. For closed-shell molecules values between $10 \%$ and $15 \%$ are more typical, but the TER is often higher in open-shell than in closed-shell molecules. For comparison values around $20 \%$ were observed for the statistical dissociation of allyl ${ }^{55,56}$ and propargyl ${ }^{57}$ radicals after UV excitation. Again we should also turn to benzyl for comparison: H-atom Rydberg time-of flight spectroscopy of benzyl, photoexcited between 228 and $270 \mathrm{~nm}$ into the D-band, yielded $\left\langle f_{T}\right\rangle \approx 0.29,{ }^{17}$ while translational energy spectroscopy at $248 \mathrm{~nm}$ yielded $\left\langle f_{T}\right\rangle \approx 0.2,{ }^{16}$ i.e., comparable values to the ones observed here at $250 \mathrm{~nm}$. No data are yet available for photodissociation of benzyl from the $\mathrm{D}_{3}$ state at $305 \mathrm{~nm}$. It is well known that the energy in the reverse barrier is predominantly released into translation. ${ }^{58}$ However, while in the case of pathway (3), the reverse barrier of 0.85 $\mathrm{eV}$ might contribute to a comparably large $\left\langle f_{T}\right\rangle$, there is no reverse barrier computed for pathway (1). Thus, this reaction should be close to an ideal statistical one and a smaller TER is expected.

The second striking observation is the fast rates for $\mathrm{H}$ atom loss derived in the experiments, with $\mathrm{k}_{H}$ on the order of several $10^{7} \mathrm{~s}^{-1}$ or more. RRKM computations yielded rate constants that were several orders of magnitude slower around $310 \mathrm{~nm}$. The small contribution of $\mathrm{H}$-atoms from multiphoton excitation cannot explain this discrepancy. Interestingly, in the case of benzyl, measured rate constants were also at least one order of magnitude faster than computed ones. ${ }^{17}$ This indicates a systematic effect present in all three radicals (benzyl, ortho-, and para-xylyl). Dissociation from the xylyl A-state has to be considered as a pathway. The $P\left(E_{T}\right)$ reveals that products are formed in their electronic ground states, and the isotropic $\mathrm{H}$-atom photofragment angular distribution rules out a direct excited state dissociation. On the other hand electronic spectra of the radicals show a number of long-lived bands with lifetimes of some $100 \mathrm{~ns},{ }^{24}$ indicating a bound excited state potential that does not dissociate to products in their electronic ground state.

Thus the experimental data are in agreement with a dissociation on the ground state potential energy surface, but energy randomization seems to be incomplete and phase space only partially filled. The existence of a bottleneck that separates phase space into two or more only weakly connected parts, leads to intrinsic non-RRKM behavior ${ }^{59}$ and experimental rates that deviate from the computed ones. In ethyl it was recently found that low-frequency, large amplitude motions are only weakly coupled with the higher energy modes, ${ }^{60}$ although in this case the existence of a bottleneck slowed down the reaction rate compared to computations. When we arbitrarily omitted the two (five) lowest-wavenumber modes from the RRKM computations, the rate for benzocyclobutene formation increased by one (three) orders of magnitude, while the rate for reaction (2) increased only slightly. An incompletely filled phase space can therefore explain the preponderance of reaction (3) in the dissociation of ortho-xylyl. Recent computations on benzyl found that the conical intersection between $\mathrm{D}_{1}$ and $\mathrm{D}_{0}$ lies at a ring-puckered geometry with elongated bonds, ${ }^{22}$ i.e., far away from the ground state equilibrium geometry. Assuming again a similar electronic structure for xylyl, it thus seems conceivable that a bottleneck exists and the $\mathrm{C}-\mathrm{H}$ bond breaks before the vibrational energy is completely thermalized.

\section{CONCLUSION}

The photochemistry of para- and ortho-xylyl has been investigated after UV excitation. Around $310 \mathrm{~nm}$ both radicals show a structured spectrum with origin bands at 32132 $\mathrm{cm}^{-1}$ (ortho-) and $32291 \mathrm{~cm}^{-1}$ (para). The excitation energies are accurate within $15 \mathrm{~cm}^{-1}$. Based on recent computations of benzyl it is assigned to the $\mathrm{C}^{2} \mathrm{~A}_{1}$ (3s) $\leftarrow \mathrm{X}^{2} \mathrm{~B}_{2}$ transition (in $\mathrm{C}_{2 \mathrm{v}}$ symmetry). $\mathrm{H}$-atom action spectra show bands at the same energy and thus reveal that excitation of xylyl initiates a photodissociation associated with the loss of a hydrogen atom. No H-atom signal was observed from the precursor. Both the isotropic $\mathrm{H}$-atom PAD and the translational energy release are in agreement with a dissociation in the electronic ground state that is slower than a rotational period. At $250 \mathrm{~nm}$ both xylyl isomers are excited into an intense but unstructured band. Photodissociation of para-xylyl yields para-xylylene + $\mathrm{H}$ at all wavelengths with a TER that decreases from $33 \%$ at $309.7 \mathrm{~nm}$ to $19 \%$ at $250 \mathrm{~nm}$. For ortho-xylyl a dissociation to ortho-xylylene $+\mathrm{H}$ was expected, based on computed heats of reaction. However, the $\mathrm{H}$-atom translational energy distribution is in better agreement with benzocyclobutene as the dominant photoproduct. Again, the H-atom PAD is isotropic and the translational energy release is around 30\%, assuming benzocyclobutene formation. Computationally a new pathway was found that proceeds via a scissoring motion of the $\mathrm{CH}_{2}$ and $\mathrm{CH}_{3}$ group, combined with a rotational motion of the $\mathrm{CH}_{2}$ group, which lead to simultaneous ring formation and hydrogen dissociation. It exhibits a barrier of $327 \mathrm{~kJ} / \mathrm{mol}$, much lower than the pathway identified in a previous study, which proceeds via an ortho-xylylene intermediate. Thus, the formation of benzocyclobutene becomes possible at $311.2 \mathrm{~nm}$, but due to the higher barrier and the tight transition state it should be kinetically disfavored compared with ortho-xylylene formation, as shown by RRKM calculations. Interestingly, delay scans yielded rates that are several orders of magnitude faster than expected from the RRKM calculations. This suggests that a bottleneck exists in phase space and energy is not completely randomized before dissociation, which might explain the formation of benzocyclobutene.

\section{SUPPLEMENTARY MATERIAL}

See supplementary material for further experimental and computational data. 


\section{ACKNOWLEDGMENTS}

Financial support by the Deutsche Forschungsgemeinschaft through Contract No. FI575/8-2 is gratefully acknowledged. This project was also supported by the Swiss Federal Office of Energy (SFOE, Contract No. SI/501269-01) and by the Laboratory for Thermal Processes and Combustion (LTV) located at PSI. We would like to thank Stefan Müller and Nicolas Scheuplein for their contributions to the experiment.

${ }^{1}$ G. da Silva, C.-C. Chen, and J. W. Bozzelli, J. Phys. Chem. A 111, 8663 (2007).

${ }^{2}$ P. Hemberger, A. J. Travitt, T. Gerber, E. Ross, and G. da Silva, J. Phys. Chem. A 118, 3593 (2014).

${ }^{3}$ T. W. Schmidt, Int. Rev. Phys. Chem. 35, 209 (2016).

${ }^{4}$ D. K. Hahn, S. J. Klippenstein, and J. A. Miller, Faraday Discuss. 119, 79 (2002).

${ }^{5}$ I. Fischer, Chem. Soc. Rev. 32, 59 (2003).

${ }^{6}$ K. Tsukiyama and R. Bersohn, J. Chem. Phys. 86, 745 (1987).

${ }^{7}$ R. Schmiedl, H. Dugan, W. Meier, and K.-H. Welge, Z. Phys. A 304, 137 (1982).

${ }^{8}$ M. A. Oehlschlaeger, D. F. Davidson, and R. K. Hanson, J. Phys. Chem. 110, 6649 (2006).

${ }^{9}$ D. C. Astholz and J. Troe, J. Chem. Soc., Faraday Trans. 2 78, 1413 (1982).

${ }^{10}$ W. Mueller-Markgraf and J. Troe, J. Phys. Chem. 92, 4899 (1988).

${ }^{11}$ H. Hippler, C. Reihs, and J. Troe, Z. Phys. Chem. 167, 1 (1990).

${ }^{12}$ J. Jones, G. B. Bacskay, and J. C. Mackie, J. Phys. Chem. A 101, 7105 (1997).

${ }^{13}$ M. Braun-Unkhoff, P. Frank, and T. Just, Ber. Bunsengesellschaft Phys. Chem. 94, 1417 (1990).

${ }^{14}$ G. T. Buckingham, T. K. Ormond, J. P. Porterfield, P. Hemberger, O. Kostko, M. Ahmed, D. J. Robichaud, M. R. Nimlos, J. W. Daily, and G. B. Ellison, J. Chem. Phys. 142, 044307 (2015).

${ }^{15}$ G. T. Buckingham, J. P. Porterfield, O. Kostko, T. P. Troy, M. Ahmed, D. J. Robichaud, M. R. Nimlos, J. W. Daily, and G. B. Ellison, J. Chem. Phys. 145, 014305 (2016)

${ }^{16}$ M. Shapero, N. C. Cole-Filipiak, C. Haibach-Morris, and D. M. Neumark, J. Phys. Chem. A 119, 12349 (2015).

${ }^{17}$ Y. Song, X. Zheng, M. Lucas, and J. Zhang, Phys. Chem. Chem. Phys. 13, 8296 (2011).

${ }^{18}$ G. da Silva, J. A. Cole, and J. W. Bozelli, J. Phys. Chem. A 114, 2275 (2010).

${ }^{19}$ G. da Silva, J. A. Cole, and J. W. Bozzelli, J. Phys. Chem. A 113, 6111 (2009).

${ }^{20}$ C. Cavallotti, M. Derudi, and R. Rota, Proc. Combust. Inst. 32, 115 (2009).

${ }^{21}$ M. Zierhut, B. Noller, T. Schultz, and I. Fischer, J. Chem. Phys. 122, 094302 (2005).

${ }^{22}$ A. Röder, A. Humeniuk, J. Giegerich, I. Fischer, L. Poisson, and R. Mitric, Phys. Chem. Chem. Phys. 19, 12365 (2017).

${ }^{23}$ M. Margraf, B. Noller, C. Schroter, T. Schultz, and I. Fischer, J. Chem. Phys. 133, 074304 (2010).

${ }^{24}$ T. R. Charlton and B. A. Thrush, Chem. Phys. Lett. 125, 547 (1986).

${ }^{25}$ T.-Y. D. Lin, X.-Q. Tan, T. M. Cerny, J. M. Williamson, D. W. Cullin, and T. A. Miller, Chem. Phys. 167, 203 (1992).

${ }^{26}$ J. I. Selco and P. G. Carrick, J. Mol. Spectrosc. 173, 277 (1995).

${ }^{27}$ T. Bierkandt, P. Hemberger, P. Osswald, M. Koehler, and T. Kasper, Proc. Combust. Inst. 36, 1223 (2017).

${ }^{28}$ S. Gudiyella, T. Malewicki, A. Comandini, and K. Brezinsky, Combust. Flame 158, 687 (2011).
${ }^{29}$ P. Dagaut and S. Gail, Combust. Sci. Technol. 179, 813 (2007).

${ }^{30}$ R. H. Natelson, M. S. Kurmann, R. O. Johnson, P. Cernansky, and D. L. Miller, Combust. Sci. Technol. 183, 897 (2011).

${ }^{31}$ Y. Murakami, T. Oguchi, K. Hashimoto, and Y. Nosaka, J. Phys. Chem. A 113, 10652 (2009).

${ }^{32}$ F. Battin-Leclerc, R. Bounaceur, N. Belmekki, and P. A. Glaude, Int. J. Chem. Kinet. 38, 284 (2006).

${ }^{33}$ G. da Silva, E. E. Moore, and J. W. Bozzelli, J. Phys. Chem. A 113, 10264 (2009).

${ }^{34}$ Y. Li, L. Zhang, T. Yuan, K. Zhang, J. Yang, B. Yang, F. Qi, and C. K. Law, Combust. Flame 157, 143 (2010).

${ }^{35}$ L. Zhao, Z. Cheng, L. Ye, F. Zhang, L. Zhang, F. Qi, and Y. Li, Proc. Combust. Inst. 35, 1745 (2015).

${ }^{36}$ P. Hemberger, A. J. Travitt, E. Ross, and G. da Silva, J. Phys. Chem. Lett. 4, 2546 (2013).

${ }^{37}$ A. T. J. B. Eppink and D. H. Parker, Rev. Sci. Instrum. 68, 3477 (1997).

${ }^{38}$ Imaging in Molecular Dynamics, edited by B. J. Whitaker (Cambridge University Press, Cambridge, 2003).

${ }^{39}$ M. N. R. Ashfold, N. H. Nahler, A. J. Orr-Ewing, O. P. J. Vieuxmaire, R. L. Toomes, T. N. Kitsopoulos, I. A. Garcia, D. A. Chestakov, S. M. Wu, and D. H. Parker, Phys. Chem. Chem. Phys. 8, 26 (2006).

${ }^{40} \mathrm{~J}$. Giegerich, I. Fischer, J. Petersen, and R. Mitric, Phys. Chem. Chem. Phys. 16, 6294 (2014).

${ }^{41}$ M. S. Schuurman, J. Giegerich, K. Pachner, D. Lang, B. Kiendl, R. J. MacDonell, A. Krueger, and I. Fischer, Chem. Eur. J. 21, 14486 (2015).

${ }^{42}$ M. Steinbauer, J. Giegerich, K. H. Fischer, and I. Fischer, J. Chem. Phys. 137, 014303 (2012).

${ }^{43}$ W. Noyes, Organic Synthesis Collection (Wiley, New York, 1943), Vol. 2.

${ }^{44}$ G. Garcia, L. Nahon, and I. Powis, Rev. Sci. Instrum. 75, 4989 (2004).

${ }^{45}$ M. Fukushima and K. Obi, J. Chem. Phys. 93, 8488 (1990).

${ }^{46}$ N. Ikeda, N. Nakashima, and K. Yoshihara, J. Phys. Chem. 88, 5803 (1984).

${ }^{47}$ G. Porter and F. J. Wright, Trans. Faraday Soc. 51, 1469 (1955).

${ }^{48}$ C. M. Western, J. Quant. Spectrosc. Radiat. Transfer 186, 221 (2016).

${ }^{49}$ D. J. Clouthier and D. A. Ramsay, Annu. Rev. Phys. Chem. 34, 31 (1983).

${ }^{50}$ J. C. D. Brand, J. Chem. Soc. 1956, 858.

${ }^{51}$ J. M. Bowman and X. Zhang, Phys. Chem. Chem. Phys. 8, 321 (2006).

${ }^{52}$ S. W. North, A. J. Marr, A. Furlan, and G. E. Hall, J. Phys. Chem. A 101, 9224 (1997).

${ }^{53}$ M. J. Frisch, G. W. Trucks, H. B. Schlegel, G. E. Scuseria, M. A. Robb, J. R. Cheeseman, G. Scalmani, V. Barone, B. Mennucci, G. A. Petersson, H. Nakatsuji, M. Caricato, X. Li, H. P. Hratchian, A. F. Izmaylov, J. Bloino, G. Zheng, J. L. Sonnenberg, M. Hada, M. Ehara, K. Toyota, R. Fukuda, J. Hasegawa, M. Ishida, T. Nakajima, Y. Honda, O. Kitao, H. Nakai, T. Vreven, J. A. Montgomery, Jr., J. E. Peralta, F. Ogliaro, M. J. Bearpark, J. Heyd, E. N. Brothers, K. N. Kudin, V. N. Staroverov, R. Kobayashi, J. Normand, K. Raghavachari, A. P. Rendell, J. C. Burant, S. S. Iyengar, J. Tomasi, M. Cossi, N. Rega, N. J. Millam, M. Klene, J. E. Knox, J. B. Cross, V. Bakken, C. Adamo, J. Jaramillo, R. Gomperts, R. E. Stratmann, O. Yazyev, A. J. Austin, R. Cammi, C. Pomelli, J. W. Ochterski, R. L. Martin, K. Morokuma, V. G. Zakrzewski, G. A. Voth, P. Salvador, J. J. Dannenberg, S. Dapprich, A. D. Daniels, Ö. Farkas, J. B. Foresman, J. V. Ortiz, J. Cioslowski, and D. J. Fox, gaussian 09, Revision B.01, Gaussian, Inc., Wallingford, CT, USA, 2009.

${ }^{54}$ M. Abe, Chem. Rev. 113, 7011 (2013).

${ }^{55}$ H.-J. Deyerl, I. Fischer, and P. Chen, J. Chem. Phys. 110, 1450 (1999).

${ }^{56}$ Y. Song, M. Lucas, M. Alcaraz, J. Zhang, and C. Brazier, J. Phys. Chem. A 119, 12318-12328 (2015).

${ }^{57}$ H.-J. Deyerl, I. Fischer, and P. Chen, J. Chem. Phys. 111, 3441 (1999).

${ }^{58}$ D. H. Mordaunt, D. L. Osborn, and D. M. Neumark, J. Chem. Phys. 108, 2448 (1998)

${ }^{59}$ T. Baer and W. L. Hase, Unimolecular Reaction Dynamics (Oxford University Press, New York, 1996).

${ }^{60}$ A. Bach, J. M. Hostettler, and P. Chen, J. Chem. Phys. 123, 021101 (2005). 